\title{
HOMOTOPY LIMITS AND THE HOMOTOPY TYPE OF FUNCTOR CATEGORIES
}

\author{
DAVID A. COX
}

\begin{abstract}
Let Y: $I \rightarrow$ Simplicial Sets be a functor. We give a sufficient condition for the map holim $\mathbf{Y} \rightarrow \lim \mathbf{Y}$ to be a weak equivalence. Then we apply this to determine the Artin-Mazur homotopy type of the functor category Funct(I, Sets).
\end{abstract}

1. Homotopy direct limits. Let $I$ be a small category, and let $\mathbf{Y}: I \rightarrow \delta$ be a functor ( $\delta$ is the category of simplicial sets). In [3, XII 2.1 and 3.7], Bousfield and Kan define holim Y, the homotopy direct limit of $\mathbf{Y}$ (Bousfield and Kan work with the pointed category $\delta_{*}$, but they remark [3, XII 3.7] that everything remains true in the unpointed case). They also construct a natural map holim $\mathbf{Y} \rightarrow \lim \mathbf{Y}$. Proposition 1 below gives a sufficient condition for this map to $\vec{b}$ e a weak $\overrightarrow{\text { equivalence. }}$

First, we need some notation. The "underlying space" or "nerve" of a small category $I$ is denoted $N(I)$ (this differs from the notation of [3, XI 2.1]). If $i$ is an object of $I$, then $I \backslash i$ is the category of all maps $i \rightarrow j$ in $I$ (see [3, XI 2.7]), and we have the formula:

$$
N(I \backslash i)_{q}=\coprod_{u \in N(I)_{q}} \operatorname{Hom}_{I}\left(i, i_{q}\right), \quad u=\left(i_{0} \leftarrow \cdots \leftarrow i_{q}\right) .
$$

And given a functor $\mathbf{Y}: I \rightarrow \delta$, we get the functors $\mathbf{Y}_{n}: I \rightarrow$ Sets (for $n \geqslant 0$ ), which are defined as follows: if $i$ is an object of $I$, then $\mathbf{Y}_{n}(i)$ is just $\mathbf{Y}(i)_{n}$, the $n$-simplices of $\mathbf{Y}(i)$.

Proposition 1. Let $\mathbf{Y}: I \rightarrow \delta$ be a functor, and assume that each $\mathbf{Y}_{n}$ is a coproduct of representable functors. Then the natural map:

$$
\underset{\rightarrow}{\operatorname{holim}} \mathbf{Y} \rightarrow \lim _{\rightarrow} \mathbf{Y}
$$

is a weak equivalence.

Proof. By assumption, each $\mathbf{Y}_{n}$ can be written as:

$$
\mathbf{Y}_{n}=\coprod_{\alpha \in A_{n}} \operatorname{Hom}_{I}\left(i_{\alpha},\right)
$$

Received by the editors July 8, 1975.

AMS (MOS) subject classifications (1970). Primary 14F35, 55D99, 18G30; Secondary 14F20, $18 \mathrm{~F} 10$.

Key words and phrases. Simplicial set, homotopy direct limit, functor category, Artin-Mazur homotopy type, hypercovering, nerve of a category. 
where $A_{n}$ is some set. Since the direct limit of any representable functor is a singleton set, we see that $(\lim \mathbf{Y})_{n}=\lim \mathbf{Y}_{n} \simeq A_{n}$.

Next let us look at holim $\vec{Y}$. We define the bisimplicial set $Y$.. as follows:

$$
Y_{\cdot q}=\coprod_{u \in N(I)_{q}} \mathbf{Y}\left(i_{q}\right), \quad u=\left(i_{0} \leftarrow \cdots \leftarrow i_{q}\right)
$$

where the boundaries and degeneracies are as in [3, XII 5.1] (and Y.. is called $\left.\amalg_{*} \mathbf{Y}\right)$. Then, by [3, XII 5.2], we have:

$$
\underset{\rightarrow}{\operatorname{holim}} \mathbf{Y}=\operatorname{diag}\left(Y_{. .}\right) \text {. }
$$

Using (4), (3) and (1), we obtain:

$$
\begin{aligned}
Y_{p q} & =\coprod_{u \in N(I)_{q}} \mathbf{Y}_{p}\left(i_{q}\right)=\coprod_{u \in N(I)_{q}} \coprod_{\alpha \in A_{p}} \operatorname{Hom}_{I}\left(i_{\alpha}, i_{q}\right) \\
& =\coprod_{\alpha \in A_{p}} \coprod_{u \in N(I)_{q}} \operatorname{Hom}_{I}\left(i_{\alpha}, i_{q}\right)=\coprod_{\alpha \in A_{p}} N\left(I \backslash i_{\alpha}\right)_{q}
\end{aligned}
$$

and one sees easily that $Y_{p}=\coprod_{\alpha \in A_{p}} N\left(I \backslash i_{\alpha}\right)$.

Let $W$. be the bisimplicial set where $W_{p q}=(\lim \mathbf{Y})_{p} \simeq A_{p}$ (so that $W_{p}$. $\simeq \amalg_{\alpha \in A_{p}}{ }^{*}, *$ being the trivial simplicial set). The obvious maps $N\left(I \backslash i_{\alpha}\right) \rightarrow *$ give us maps:

$$
Y_{p} .=\coprod_{\alpha \in A_{p}} N\left(I \backslash i_{\alpha}\right) \rightarrow \coprod_{\alpha \in A_{p}} *=W_{p}
$$

so that we have a bisimplicial map $Y_{. .} \rightarrow W$. . And we see that the map (2) above is just the map diag $\left(Y_{. .}\right) \rightarrow \operatorname{diag}\left(W_{.}\right)$. So we need to prove that it is a weak equivalence.

By [3, XII 3.4], we have a commutative diagram:

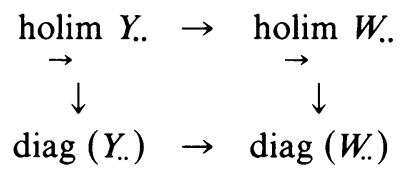

where the vertical arrows are weak equivalences. But each $N\left(I \backslash i_{\alpha}\right)$ is contractible (see [3, XI 2.4]), so that the maps (6) are weak equivalences. Thus, [3, XII 4.2] says that the top arrow of (7) is a weak equivalence, which forces the bottom arrow to be one too. Q.E.D.

2. The homotopy type of a functor category. As before, let $I$ be a small category. We want to determine the Artin-Mazur homotopy type of the functor category $\operatorname{Funct}\left(I\right.$, Sets) (which we will denote $I^{\wedge}$; see [1, I 1.2]).

What does it mean for $I^{\wedge}$ to have a homotopy type? Looking at $[2, \S 9]$, we see that we need the following two things for $I^{\wedge}$ : a Grothendieck topology and a connected component functor.

The first of these is easy to describe: a map $f: F \rightarrow G$ in $\bar{I}^{\wedge}$ is a covering iff for all $i$ in $I, f(i)$ is onto (this is the canonical topology of the topos $I^{\wedge}[1$, IV 2.6]).

If one analyzes the connected component functor of $[2, \S 9]$, one sees that it is nothing but a left adjoint to the constant sheaf functor (see [1, IV 7.6 and 
8.7] for full details). But in the case of $I^{\wedge}$, a constant sheaf is just a constant functor on $I$, and the left adjoint is well known to be direct limit, lim.

Let $H R\left(I^{\wedge}\right)$ be the homotopy category of hypercoverings of $I^{\wedge}$ (see $[2,8.4$ and 8.13]). Then, following [2, \$9], we have:

Definition. The homotopy type of $\hat{I^{\hat{n}}}$, denoted $\left\{\hat{I^{\hat{n}}}\right\}_{h t}$, is the pro-object $\left\{\lim _{\rightarrow} U .\right\}_{U . \in H R\left(I^{\prime}\right)}$ in pro- $\mathcal{H}$ (where $\mathcal{H}$ is the homotopy category).

PROPOSITION 2. There is a canonical isomorphism:

$$
\left\{I^{\wedge}\right\}_{h t} \simeq N(I)
$$

in pro- $\mathcal{H}$.

Proof. First, note that a simplicial object $U$. of $I^{\wedge}$ can be regarded as a functor $U: I \rightarrow \delta$ and then $\lim U$. has the same meaning it had in $\S 1$.

We will use $e$. to denote the trivial simplicial object of $I^{\wedge}$ (each $e_{n}$ is the functor which takes all of $I$ to the same one element set). Note that for any simplicial object $U$. of $I^{\wedge}$, there is a unique map $U$. $\rightarrow e$.

Let $U$. be a hypercovering of $I^{\wedge}$. Then, by [2, 8.5(a)], each $U .(i)$ (for $i$ in $I$ ) is a contractible Kan complex, so that for each $i$ in $I$, the map $U .(i) \rightarrow e .(i)$ is a weak equivalence. Then, by [3, XII 4.2], the map:

$$
\underset{\rightarrow}{\operatorname{holim}} U . \underset{\rightarrow}{\operatorname{holim}} e .
$$

is a weak equivalence. Formulas (4) and (5) show that holim $e$. is just $N(I)$, so that we get a weak equivalence holim $U . \simeq N(I)$.

Next, assume that each $U_{n}$ is a $\overrightarrow{\text { coproduct }}$ of representable functors. Then, by Proposition 1, the map:

$$
\underset{\rightarrow}{\operatorname{holim}} U . \rightarrow \lim _{\rightarrow} U .
$$

is a weak equivalence. So for such $U$.'s, there is a canonical weak equivalence $\lim U \simeq N(I)$. If we can show that these $U$ 's are cofinal in $H R\left(I^{\wedge}\right)$, then the

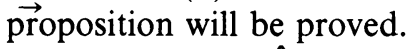

For any $F$ in $I^{\wedge}$ and $i$ in $I$, there is a natural transformation of functors $F(i) \times \operatorname{Hom}_{I}(i,) \rightarrow F \quad$ (where the pair $(x, f) \in F(i) \times \operatorname{Hom}_{I}(i, j)$ gets mapped to $F(f)(x) \in F(j))$. Putting these together, we get a map:

$$
\coprod_{i \in o b I} F(i) \times \operatorname{Hom}_{I}(i,) \rightarrow F
$$

which is a covering in $I^{\wedge}$. This shows that anything in $I^{\wedge}$ can be covered by a coproduct of representable functors. Then, using the techniques of $[2, \S 8]$, one easily proves that hypercoverings with the above property are cofinal in $H R\left(I^{\wedge}\right)$. Q.E.D.

REMARK. One can also do a pointed version of this. Let $i_{0}$ be a fixed object of $I$. We get a point of $I^{\wedge}$ (in the sense of $[2, \S 8]$ ), $p: I^{\wedge} \rightarrow$ Sets, defined by the formula $p(F)=F\left(i_{0}\right)$. Then we get the pointed homotopy type of $I^{\wedge}$, still denoted $\left\{I^{\wedge}\right\}_{h t}$, in pro- $\mathcal{H}_{*}$, which turns out to be just $N(I)$ with $i_{0}$ as the distinguished vertex. 


\section{REFERENCES}

1. M. Artin, A. Grothendieck and J. L. Verdier, Théorie des topos et cohomologie étale des schémas. Tome 1: Théorie des topos, (SGA 4), Lecture Notes in Math., vol. 269, Springer-Verlag, Berlin and New York, 1972. MR 50 \#1130.

2. M. Artin and B. Mazur, Etale homotopy, Lecture Notes in Math., vol. 100, Springer-Verlag, Berlin and New York, 1969. MR 39 \#6883.

3. A. K. Bousfield and D. M. Kan, Homotopy limits, completions and localizations, Lecture Notes in Math., vol. 304, Springer-Verlag, Berlin and New York, 1972.

Department of Mathematics, Haverford College, Haverford, Pennsylvania 19041

Current address: Department of Mathematics, Hill Center, Rutgers University, New Brunswick, New Jersey 08903 